\title{
Entrelacs
}

ENTRELACS Cinéma et audiovisuel

$10 \mid 2013$

Le Toucher

\section{La Corde, pour un dessin filmique}

Daphné Le Sergent

\section{OpenEdition}

Journals

Édition électronique

URL : http://journals.openedition.org/entrelacs/477

DOI : 10.4000/entrelacs.477

ISSN : 2261-5482

\section{Éditeur}

Éditions Téraèdre

\section{Référence électronique}

Daphné Le Sergent, «La Corde, pour un dessin filmique », Entrelacs [En ligne], 10 | 2013, mis en ligne le 10 septembre 2013, consulté le 22 avril 2019. URL : http://journals.openedition.org/entrelacs/477 ; DOI : 10.4000/entrelacs.477

Ce document a été généré automatiquement le 22 avril 2019

Tous droits réservés 


\title{
La Corde, pour un dessin filmique
}

\author{
Daphné Le Sergent
}

1 1948. La Corde d'Alfred Hitchcock. Le rideau de la fenêtre s'ouvre. Un visage tordu de douleur. Deux hommes qui l'étranglent. Le cadre nous fait pénétrer dans un appartement bourgeois et nous dévoile le secret de ce meurtre, le cadavre de la victime venant d'être dissimulé dans un grand coffre au milieu de la pièce. Deux jeunes hommes ont assassiné un de leurs camarades à l'aide d'une corde. À présent ils se préparent à accueillir des invités pour une fête où secrètement ils pensent savourer leur crime. En effet, ce meurtre leur semble " parfait », à l'égal d'un geste artistique, gratuit, non dicté par un mobile mais par l'élégance seule de son illusion philosophique. Ne pas faire cette fête, remarque amèrement l'un des deux, reviendrait à réaliser un tableau sans l'accrocher. D'ailleurs un nombre conséquent de peintures jonche les murs de l'appartement, notamment une toile proche de l'expressionnisme abstrait portée rapidement à la plaisanterie par l'une des convives (« ma jeune filleule de trois ans est capable des mêmes prouesses »).

2 Le film semble vouloir habiller ce geste de strangulation en se composant selon une structure théâtrale $^{1}$ : unité de temps, de lieu et d'action. Le décor est une immense fenêtre donnant sur la ville de New-York, une maquette indiquant le moment de la journée, soit qu'elle bénéficie d'un éclairage blanc diurne, soit qu'elle brille du feu nocturne des lumières artificielles, véritable palette du premier film en couleur du cinéaste. Au fil de cette tapageuse célébration macabre, certains détails intriguent l'un des invités, un ancien professeur à eux. Partant de signes qui pourraient être anodins pour arriver à des indices d'un comportement inhabituel, celui-ci remonte jusqu'aux coupables et les démasque à la fin de la soirée. Le spectateur, ayant depuis le début suivi les agissements des criminels, peut apprécier l'enquête et se laisser porter par le fil de l'intrigue qui se délie progressivement avec leur arrestation finale. Le film se déroule comme un seul long plan-séquence, illusion d'une unique trajectoire de la caméra dans cet espace clos. Une main conduit le cadre à se focaliser sur un verre, une silhouette ramène les regards à la table, un plan d'ensemble invite à faire un pas en arrière. De cette manière, le spectateur est incité à glisser entre les personnages, comme s'il se tenait luimême dans l'espace diégétique. Ce plan-séquence s'étire et confère l'impression d'un 
couloir visuel. Celui-ci pourrait être comparé à une ligne ou à une sorte de serpent, qui va de corps en corps, s'étrangle pour raccorder, s'allonge afin d'entretenir le suspense, parfois tapi dans un coin du décor comme pour "guetter sa proie», et s'emparer du prochain indice donné en pâture. Plus ce couloir visuel - cette présumée ligne - s'étend en durée et en profondeur, plus il entraîne dans son cours des preuves accablantes et en précipite l'issue, la découverte du crime et de son châtiment. Le trajet que suit le regard du spectateur ressemble ainsi à une corde glissant tout au long de l'intrigue jusqu'au dénouement, jusqu'à ce que se resserre l'étau des soupçons sur les deux jeunes hommes.

3 Fin des années 40. Début des premiers drippings de Pollock, projections de coulures sur la toile, lignes sinueuses et entrelacs colorés. Avec ces drippings, le travail de la main laisse place au geste et à toute l'impulsion du corps. Cela relève d'une implication physique à l'intérieur de la toile, devenue "arène " pour Harold Rosenberg. Dorénavant, c'est le corps tout entier qui s'affirme dans l'œuvre. Il ne s'agit pas d'un point de vue issu d'une représentation, du sommet fixe de la pyramide perspectiviste, mais d'un point en perpétuel mouvement glissant au sein du tableau posé au sol. Action Painting formulait Rosenberg. Tout réside dans le vertige d'un «ici, maintenant», d'un geste qui inscrit l'être dans son œuvre, augurant un possible ancrage dans le monde.

4 Avec Pollock, c'est une nouvelle conception du dessin qui se met en place, non plus ligne apposée sur une surface plane mais trace d'un déplacement, empreinte d'un mouvement. Le dessin n'est plus donné comme contour des choses, fermeture de la forme et gage de la représentation de l'Ut Pictura Poesis. Il ne semble plus distribuer le sens sur le plan du tableau. La ligne n'est plus optique mais haptique ${ }^{2}$. Elle n'est plus tant émerveillement purement rétinien que parcours de l'œil le long des formes, caresse dont la trajectoire met en contact l'un et l'autre, l'image du monde et le dynamisme du regard, extériorité du réel et intériorité vécue du spectateur. En ce sens, elle relève du toucher.

5 L'art de la première moitié du 20ème siècle s'est déjà emparé de cet haptisme, de cette ligne qui n'est pas assujettie à une représentation mais à une sensation (Paul Klee, Kandinsky). Pour les artistes, la ligne haptique est la ligne moderne, elle nous plonge dans le monde des choses $^{3}$ à l'instar, pour Jean Epstein, du gros plan cinématographique. Elle annule toute distance entre le sujet et l'image en ce qu'elle propose une projection cinétique du regard du spectateur, invité à suivre des lignes courbes, droites, à sauter de couleur en couleur. « Dans l'œuvre d'art, note Paul Klee, les chemins sont ménagés à cet œil du spectateur en train d'explorer comme un animal pâture une prairie. $»^{4}$ L'espace cubiste, quant à lui, n'obéit plus aux lois perspectivistes impliquant une hiérarchisation $\mathrm{du}$ proche et du lointain par une mise en scène mais subit un éclatement, une fragmentation, que l'œil haptique traverse. «Tout se passe, écrit Jean Paulhan, comme si notre regard n'était plus qu'une allonge à nos doigts, une antenne à notre front $»^{5}$.

6 Le regard est mouvement, il a abandonné le sommet de la pyramide perspectiviste, ce «cogito» de l'image, ce point de vue réflexif sur le monde, pour se plonger dans le cinétisme ininterrompu des choses. Charles Lapicque, critique d'art des années cinquante, écrit au sujet de l'art moderne : « En peinture, [...] le visible se congèle, l'image devient la chose. [...] J'imagine que j'approche, que je touche même la table, tandis que dans le même temps je reste toujours aussi loin d'elle, cloué que je suis au centre perspectif du tableau. L'apparence refuse de se multiplier, c'est moi qui me divise. $»^{6}$ Avec la conception haptique de l'art, le regard est conçu comme force de projection tout autant qu'entraînant une division, une schize dans le sujet. Déjà, face au cinéma, Walter Benjamin évoquait la «distraction» du spectateur, le fait qu'il soit coupé de lui-même ${ }^{7}$. 
Pourrait-on déceler, avec l'haptisme, un nouveau régime de visibilité où se joue une dissociation, écartèlement du sujet entre le « là-bas » du regard et le « ici » du toucher, dans l'hétérogène d'un réel qu'il «touche » des yeux ? Est-ce qu'en ce sens on pourrait penser conjointement le champ des arts plastiques et celui du cinéma? Si La Corde d'Hitchcock fait montre d'une ligne particulière dans le mouvement de la caméra, en quoi cela rejoint-il les préoccupations des artistes ? A une époque où figuration et abstraction sont peu à peu évacuées de l'avant-garde au profit de la performance ou de l'installation, est-il encore juste de parler de ligne ou de dessin? Est-ce qu'un dessin filmique, comparable à la ligne haptique de l'art moderne, pourrait y être révélé ? En quoi la ligne haptique serait-elle à même de rendre compte de la réalité ? C'est dans un dialogue constant entre le champ des arts plastiques et celui du cinéma que nous proposons d'aborder cette réflexion.

\section{La plénitude des choses}

7 Tout d'abord, comment comprendre l'établissement de cet interminable plan-séquence dans le film d'Hitchcock? Dans quelle mesure le cinéaste cherche-t-il à susciter un dessin, entendu ici comme ensemble de traits visant à caractériser de façon haptique une chose? Avant tout, il nous faut remarquer l'attachement du cinéaste pour le domaine des arts : les cours d'histoire de l'art, de dessin et de peinture qu'il suit en 1915, les débuts de sa carrière en tant que graphiste puis comme dessinateur d'intertitres de films, et enfin l'apprentissage de son métier passant, entre autres, par le dessin des films plan par plan. Hitchcock est un grand amateur de peintures. Suite à La maison du docteur Edwardes (1945), dans lequel il fait appel à Dalí pour réaliser le décor de la scène du rêve, il entreprend avec son épouse une collection d'œuvres d'art. « Pour moi, rapporte-t-il, un film doit être planifié sur le papier. [...] Pour cela, on doit posséder un sens visuel. Je ne regarde jamais avec la caméra ; je pense seulement à un écran blanc, qui doit être rempli comme on peint une toile. C'est pourquoi je dessine des plans grossiers pour le cadreur. $»^{8}$

Dans La Corde, rien n'est laissé au hasard. Le film se déroule tel un tableau vivant puisque la contrainte technique du plan-séquence oblige acteurs et caméraman à se mouvoir selon un plan établi à l'avance (nécessitant avant le tournage dix jours de répétition). Le mot de "performance artistique " pourrait alors s'imposer en lien avec l'idée d'action painting chez Pollock ou encore, au regard de cette même année 1948, aux travaux de John Cage et Merce Cunningham ${ }^{9}$. Mais pour ces artistes, la question de la performance se tient bien loin de la maitrise hitchckockienne puisque s'y jouent les questions d'improvisation, un geste qui ne soit ni relié à un devoir de figuration, ni à celui d'une partition ou d'une chorégraphie préparée à l'avance.

Si nous devions rapprocher le style d'Hitchcock à un mouvement ou à un style artistique, ce serait pour Jacques Aumont celui, dix-neuvièmiste, du symbolisme. Tout d'abord, le recours à des images oniriques (La maison du docteur Edwardes) ou à des obsessions inconscientes (Vertigo, 1958), met en place une dimension cachée, mystérieuse mais latente, derrière le monde réel, et pourrait trouver héritage dans le fantastique et l'onirisme des peintures de ce courant. D'autre part, il y a dans l'œuvre hitchcockienne, une prime importance accordée aux objets, pouvant être pris dans les apparences discrètes de cet énigmatique revers. Mais, pour Jacques Aumont, c'est ici que s'arrête la comparaison avec le symbolisme, puisque ces objets ne sont pas tant compris dans leur effet de signification ou de symbolisation que dans la traduction directe du réel, dans sa 
quasi-littéralité. "L'image symboliste est l'image d'un immatériel, d'un invisible, d'un idéal, d'une Idée. [...] Hitch (sic) a parfaitement reconnu que le cinéma n'est pas une allégorisation mais une interprétation du monde, et que, si les images ont un pouvoir de symbolisation (il faut les construire dans cette visée) ce n'est pas un pouvoir métaphorique mais un pouvoir figural et en même temps réaliste (autre paradoxe). $»^{10}$ Ces objets se présentent à nous dans une transfiguration du monde mais celle-ci n'est en rien idéelle, il s'agirait plus d'un "étonnement» dû à la vie qui les anime. Jacques Aumont prend pour exemple les lieux en hauteur : falaise, clocher, balcon, statue, qui font naître des sensations chez les personnages mais aussi, par le travail de l'image, chez le spectateur. D'où la présence du figural chez Hitchcock, que Philippe Dubois définit comme activité de vision dans l'image, dans sa configuration plastique et formelle, relevant plus de l'affect et de la sensation que du mot et du langage ${ }^{11}$. Les objets ne sont pas des symboles, la corde n'est pas tant la signification ouverte d'un désir d'ascension ou de lien mais, devenue cinématographique, elle reste objet, corde, sinuosité visuelle, planséquence. La corde nous fait toucher une corde des yeux. C'est ainsi que, de la corde qui sert à la mise à mort de la victime, nous basculons sur la corde qui sillonne de plan en plan et nous mène à l'arrestation des coupables. Le plan-séquence se teinte ainsi des propriétés de la corde-objet : usage que l'on en fait (attraper quelqu'un, serrer un corps). La corde n'est alors pas symbolique mais pragmatique, elle fait appel à l'expérience du spectateur qui connecte l'un sur l'autre le souvenir de l'objet avec la circulation dans l'espace diégétique au gré du plan-séquence.

10 Si nous décelons un dessin dans cette corde filmique, dans ce long plan-séquence, il faudrait s'appuyer sur la conception que nous en propose John Dewey. Pour lui, chaque expérience interagissant avec un objet dépose en nous l'empreinte spécifique d'un contact, une forme en adéquation avec les qualités physiques de ces choses :

Bien que le système optique puisse être isolé dans une dissection anatomique, il ne fonctionne jamais séparément. Il opère en liaison avec la main en dirigeant vers les choses et en explorant leur superficie, en guidant leur manipulation, en dirigeant la locomotion. Un tel fait a ceci pour conséquence que les qualités sensibles qui se présentent à nous au moyen du système optique sont simultanément reliées avec celles qui nous viennent des objets à travers les activités collatérales. La rondeur que l'on voit est celle des balles ; les angles perçus ne sont pas seulement le résultat de déplacements dans le mouvement des yeux, mais ce sont des propriétés des livres et des boîtes manipulées ; les courbes sont les arcs du ciel, le dôme d'un édifice ; les lignes horizontales sont vues comme la propagation de la lumière, les bords des choses autour de nous. ${ }^{12}$

11 Les lignes d'un tel dessin gardent en elles le souvenir haptique de l'expérience vécue; elles en sont la plénitude. Jackson Pollock, Robert Motherwell ou Allan Kapprow avaient redécouvert les travaux de John Dewey pour lequel l'acte de création est la mise en place d'une pratique. L'acte de création s'établit sur une expérience concrète de la vie, sur le projet de redonner leur texture charnelle aux choses, comme si leur apparence avait un avers, l'intériorité vécue. Dès lors, tout tracé que laisse le mouvement expressionniste abstrait sur la toile se teinte de la duplicité d'un je vais avec celui d'un je sens.

12 Au fond, si Hitchcock partage quelque chose avec l'avant-garde artistique américaine de cette époque-là, ce n'est en aucun cas en raison de la forme visuelle de son cinéma, ni à cause de cette idée de performance. Il s'agirait plutôt d'une même conception de l'image liée à l'expérience pragmatique de Dewey, une même impression de vertige, une impression d'être tombé dans le monde matériel des choses, de vouloir en savourer la 
substance de l'expérience, de se sentir tout contre le réel. Si ligne filmique il y a, c'est donc d'une conception toute particulière du dessin qui en découle, non assimilable à un tracé concret mais au mouvement sinueux et abstrait du regard.

Dans son épilogue à sa Théorie du film, en 1960, Siegfried Kracauer - qui s'est installé aux États-Unis - insiste sur l'effondrement des valeurs et des idéologies laissant place au champ hégémonique des sciences. Celles-ci, dans une course inépuisable au progrès technologique, dans l'interprétation matérielle qu'elles rendent du monde, dans la modélisation behaviouriste des comportements et des relations sociales, semblent avoir littéralement vidé les choses de toute l'intensité que peut leur conférer l'expérience. Le réel lui apparaît réduit à une substance mesurable, chiffrable, quantifiable, quand il n'est pas prévisible ou rattaché à une fonctionnalité. Siegfried Kracauer, inspiré par John Dewey, reconnaît ainsi dans la peinture abstraite les symptômes de la situation de l'homme moderne. "La peinture abstraite n'est pas tellement un mouvement antiréaliste qu'une révélation réaliste de l'abstraction dominante. Les compositions de lignes dont elle se délecte reflètent fidèlement la nature des processus mentaux contemporains. Comme si la peinture moderne s'attachait à faire le relevé des trajets que suivent nos pensées et nos émotions. Ces trajets ont leur correspondance dans la réalité elle-même : ils rappellent ces autoroutes et ces routes qui paraissent traverser le vide - laissant au loin des bois non frayés et des villages dérobés aux regards. $»^{13}$

Dans cette visée, en grand nombre seraient les « dessinateurs » qui tracent de leurs pas les lignes de leurs quêtes du réel : Kerouac sur la route, Humbert Humbert et Lolita en cavale, Robert Frank parcourant l'Amérique. «Je veux faire le tour de ces hautes chaînes de montagnes arides où l'on meurt de soif et de froid, de cette histoire "extratemporelle", de cet absolu du temps et de l'espace, où l'on ne trouve ni homme ni bête, ni plante où l'on va fou de solitude, avec un langage qui n'est que mots, où tout est décroché, débrayé, hors de prise avec le temps »"14 écrivait déjà Henri Miller en 1934.

Quelle pourrait être cette ligne qui dépasse le cloisonnement entre les médiums et qui semble parfois se réduire à la simple circulation des corps dans le territoire? Quelle en serait sa nature? Certes, nous l'avons évoqué, elle se joue dans un haptisme, dans une non-distance entre le sujet et le monde, soit d'un côté l'intériorité vécue du spectateur qui parcourt et de l'autre, l'extériorité du réel.

\section{Lignes haptiques}

Une autre question traversant les arts plastiques et le cinéma est celle de l'index, signe selon Charles S. Peirce ayant été en connexion physique avec son référent. Tout d'abord, il y a cette croyance documentaire qui se réclame d'une adhérence entre le temps ininterrompu du plan-séquence avec la durée de l'action réelle et qu'André Bazin théorise en réunissant index et passage. Les images cinématographiques se constituent tel un embaumement des choses (index). Parmi elles se tient le spectateur avec toute la responsabilité qui lui incombe face à la réalité et à ses ruines au sortir de la Guerre. Il leur fait face avec ce «besoin psychologique » que lui accorde Bazin où tout homme ne peut plus être dupe de la propagande. Il est impliqué dans une attitude active face au réalisme de ces images. Aussi Bazin, dans les années cinquante, admire-t-il Welles et les mouvements de caméra impliquant une profondeur de champ, tunnels creusés par le temps à la surface du visible. Tous ces passages sont ceux d'un chemin irréel, ambigu, mystérieux conférant au moment cinématographique l'épaisseur d'une durée vécue. Ces 
passages nous mènent d'un point à un autre, d'un objet à un autre, d'un visage à une histoire. Ils font glisser le regard le long d'un fil sinueux où s'égrainent les chapitres du film.

Quand, à propos de la production artistique des années soixante et soixante-dix, Rosalind Krauss utilise la notion d'index, empruntée à Peirce, elle veut dire que l'œuvre est saturée d'effets de réel, qu'à présent cette dernière ne peut plus être séparée de son contexte de monstration, que l'in situ est devenu le lot commun et l'unité de son corpus. L'index, c'est ce qui relie l'œuvre à l'espace d'exposition, les panneaux peints de Lucio Pozzi reprenant la couleur des murs de l'ancienne école où avait pris place la galerie P.S.1. (exposition Rooms, 1976, New York). L'index, chez Krauss et dans la lignée de la génération des minimalistes, c'est aussi celui de la présence pure des éléments dans l'espace. Comme face au réel, je me trouve au beau milieu des choses, présence parmi leur présence. Au fur et à mesure que j'avance dans ces installations, dans l'espace d'exposition, l'environnement m'amène à prendre conscience de la perception à jamais fluctuante que le moi a des choses, de la fragilité des rapports entre le «je » et son environnement. Aussi paraît-il naturel qu'un des ouvrages de Rosalind Krauss s'intitule « Passages ». C'est la toile de fond où se délie la notion d'index. Passage, ligne sinueuse, parcours qui nous plongent dans ces labyrinthes-œuvres.

Index. Toucher des yeux. "Je vois les yeux qui ont vu l'empereur »" écrit Roland Barthes devant la photographie du frère de Napoléon. Il y a le je touche de l'image, cet index, dont Roland Barthes n'oublie pas qu'il est lié à la croyance de celui qui voit, que les effets de sens qui en découlent s'attachent au sujet du regard, si proche du sujet d'énonciation de la linguistique. L'index est l'illusoire mise en contact entre le sujet et l'objet, il le déplace tout contre les choses, dans le sentiment de leur présence, collé à elles. ${ }^{16}$ Mais nous ne pourrions penser le tactile dans l'œuvre d'art et dans l'œuvre cinématographique sans le comprendre dans la réciprocité qu'il engage entre sentant et senti, entre le je touche et le je suis touché. Il y a, en effet, dans cette conception partagée de l'œuvre-passage la prévalence accordée au spectateur. Si nous revenons au film d'Hitchcock, le figural serait cette corde visuelle pendue au bout de nos yeux. La question du figural, au cinéma, n'est pas relative à une représentation mais à une configuration plastique de l'image, un agencement, une mise à disposition du spectateur de ses éléments - une installation serions-nous presque tentés de dire. Le figural est à l'origine de toute appréhension de l'image, de son intelligibilité et « dont Maurice Denis a donné la formule : "se rappeler qu'un tableau, avant d'être un cheval de bataille, une femme nue ou une quelconque anecdote, est essentiellement une surface plane recouverte de couleurs en un certain ordre assemblées." "Jacques Aumont, note Luc Vancheri, ne dira pas autre chose lorsque, en conclusion de ses prolégomènes à la matière, il écrit: [...]" se souvenir qu'avant d'être un drame, un document ou un blason, l'image de film est une présence visuelle, polymorphe et qui nous atteint directement." $~^{17}$ Le figural est la matière-même de l'image non pas en tant que médium - un amas de photogrammes - mais comme traversé de forces, de lignes dynamiques, de vortex ou de rythme, l'image est un souffle.

Elle est énergie lorsque les personnages qu'elle porte sont en action, elle est déchirure quand y est livrée une séparation, elle est tourbillon lorsque s'effondre le paysage, elle est calme plat si jamais la mort vient à frapper. On pense au vertige recréé par l'image hitchcockienne dans Vertigo (1958), on pense à la dissociation du corps du pickpocket, filmé par Bresson, où dans la scène du champ de course, il y a une nette coupure entre la main qui vole dans les poches et la tête immobile et placide?, entre l'acte et le jugement 
moral. On pense à L'Aurore (1927) de_Murnau où un homme va, la nuit, à la rencontre d'une femme. La caméra le suit lentement. Elle trace un long plan-séquence qui sillonne dans la rase campagne au fur et à mesure que cet homme traverse l'épaisseur sombre d'un sous-bois, dépasse une barrière, croise un chemin. Puis, sans qu'il n'y ait d'interruption dans ce plan-séquence, la caméra quitte l'homme pour couper court à travers les feuillages des arbres et venir se poster près de la femme, statique, qui l'attend et qui se remaquille. Quelques secondes passent puis l'homme rentre à nouveau dans le champ. Ils s'enlacent, la lune luit faiblement au-dessus d'eux. Le figural est cette ligne par lequel se fait le mouvement intérieur lié à la rencontre, sa sensation : le parcours où l'on rejoint l'autre, l'attente et la réunion. Aussi le figural ne fait pas exclusivement usage de lignes, de plan-séquence mais de toute la palette technique du cinéma (mouvements de caméra, montage, etc.) pour procurer une épaisseur sensible, tactile devrions-nous dire, à des réalités optiques.

Sans titre (L-beams, 1965) de Robert Morris, rassemble trois structures identiques en formes de L, positionnées chacune différemment dans l'espace d'exposition. Placées de la sorte, ces formes apparaissent étonnamment lointaines l'une de l'autre, leurs proportions respectives semblant modifiées selon que le L est couché, vertical ou bien retourné. C'est donc au fil du parcours dans l'œuvre que le spectateur engage une vision renouvelée de l'objet. S'il peut penser ces armatures comme identiques, face à l'expérience de leur apparence, il les comprend comme relationnelles, relatives à sa perception toujours mouvante. Cette installation est pour Rosalind Krauss, l'occasion de formuler un nouveau cogito : le moi n'est pas entité close, il ne préexiste pas au monde, il n'existe qu'au travers et par des moments singuliers; il se livre dans l'extériorité de l'espace public. « Nous ne sommes que la totalité de nos comportements visibles, écrit-elle, tout aussi lisibles aux autres qu'à nous-mêmes. Nos comportements sont façonnés par le monde extérieur, par ses conventions, son langage, le répertoire de ses émotions - à partir desquels nous apprenons nos propres émotions. » Il est logique que les œuvres de Morris et de Serra aient été élaborées au moment même où les romanciers français déclaraient, pour paraphraser Roland Barthes, " non point j'ai écrit, mais plutôt je suis écrit. »18

21 C'est pourquoi la question n'est pas tant celle d'un artiste qui construit sa réalisation formelle selon un plan préétabli mais celle d'un sujet affecté par le monde des choses qui l'amènent au faire. Je suis installé au beau milieu des choses, je les « ressens » mais ce jeu avec le monde est toujours éphémère, seuls demeurent les éléments qui m'ont affecté. Et l'œuvre est à présent non pas un artefact mais la présentation directe au regard du spectateur de ce qui aurait affecté l'artiste. Elle devient situation. Au même titre qu'un événement se relate selon l'énumération de ses conditions de lieu, d'intervenants ou d'objets, l'œuvre devenue situation met en place les différents éléments qui raccrochent le geste de l'artiste aux choses du monde. Les index, signes renvoyant à leur présence concrète dans le réel, semblent alors être la dissimulation de ce geste évanoui. Et l'on pourrait ajouter à la suite de Roland Barthes, je suis peint ou je suis dessiné.

Je suis touché. Film ou œuvre sont des configurations formelles d'éléments, une mise à disposition pour le passage du spectateur qui vient, en creux, se lover dans la matière. L'œuvre d'art, filmique ou appartenant aux champs des arts plastiques, est la mise en condition de cet événement sensible, l'orchestration pour l'avènement de l'affect et de la sensation $^{19}$.

23 Aussi, relativement à ce point de vue d'analyse, semble-t-il caduc de penser le médium comme matériau. Le médium serait ce jeu de réciprocité entre sentant et senti, entre je 
touche et je suis touché où ne cesse de vibrer la limite entre intérieur et extérieur qui telle une peau, ne saisit que des parcelles du réel, ne peut - contrairement à l'œil - en appréhender la totalité. Nous ne saisissons toujours que des fragments au moment-même de leur apparition. À mesure que le dessin se trace en nous, il s'évanouit. Car il n'existe qu'au travers du contact direct et immédiat des yeux avec l'image, tel un simple toucher. Comme l'annonçait Rosalind Krauss, le rapport du spectateur à l'œuvre se réalise dans un « ici, maintenant », dans l'expérience directe avec son espace concret.

Les agrégats de mouvements que rassemble la main du cinéaste pourraient-ils s'appréhender comme des gestes sans noms, sans buts, concédés au spectateur? D'un côté, le flux cinématographique s'écoule dans la succession de multiples lignes, de l'autre ces lignes le maintiennent à la lisière entre intériorité et extériorité. C'est sur cette lame que se forme le geste, geste du «toucher juste » avec le monde, geste de l'emprise sur les choses. Le dessin filmique ne semble impliquer ni un geste d'action, ni un geste effectif sur le réel. Il relèverait d'une dimension sous-jacente et sensible sur laquelle ces gestes viendraient déposer leurs empreintes, la mémoire de ce que la chose manipulée a laissé en nous.

Car si le dessin qualifié de traditionnel ou de classique a comme projet d'installer le regard à l'extérieur des choses, face aux miroitements de l'apparence, le dessin filmique cherche à projeter ces choses dans la nuit de notre intériorité. Mais il y a toujours une incertitude dans la prise, on ne sait jamais si la main ne s'est pas refermée sur du vide, alors on ouvre à nouveau les yeux. «As-tu vu le passage du vent dans les arbres, l'ombre de cet homme qui devance sa rencontre avec la femme ? - Je suis ce passage ». Le dessin filmique a tout du ruban de Mœbius : inséparabilité de sa dimension optique et du point de vue extérieur qu'elle nous confère sur les choses avec le monde intérieur des images sensibles, le tu vois et le je sens, « tu » et « je » de se confondre dans la mémoire d'un objet absent. Quand l'objet vient à disparaître, que l'objet vienne à disparaître et on se souvient. Esquisses de corps, lignes flottantes, empreintes brûlées à la surface de la feuille ou de la pellicule, la ligne haptique relate ce qu'on a vu et qui s'évanouit déjà.

\section{NOTES}

1. Le film a été tiré d'une pièce de théâtre de Patrick Hamilton.

2. Haptein en allemand : toucher

3. Aloïs Riegl, entre autres, théorise la question haptique comme relative à la proximité entre le spectateur et l'objet contemplé. En effet celui-ci, à une distance raisonnable, peut en apprécier son modelé conformément au souvenir tactile qu'il en a. Par contre, une trop grande distance implique une appréhension purement optique des formes et des choses, pouvant engendrer aberrations et fausses interprétations. (Aloïs Riegl, Grammaire historique des arts plastiques, Paris, Klincksieck, 1978.)

4. Paul Klee, Théorie de l'art moderne, Paris, Denoël, Folio/Essais, 1985, p. 38.

5. Jean Paulhan, La peinture cubiste, Paris, Gallimard, Folio/Essais, 1990, p. 29. «[...] Cet espace n'est plus, comme chez les peintres classiques, un espace qui s'enfuit devant nous jusqu'à perte de vue : c'est plutôt un espace qui s'approche, jusqu'à déborder du tableau. C'est comme un 
espace en ébullition, que le spectateur aurait charge à tout moment de maintenir - de refouler à deux mains. Cette cruche fonce sur nous de tous ses flancs. Ce fauteuil, comme on dit, nous tend les bras. Ce visage nous impose du même coup, comme à l'aveugle qui le tâte, son profil et sa face. » (Jean Paulhan, La peinture cubiste, Paris, Denoël, Folio/Essais, 1990, pp. 29-30.)

6. Charles Lapicque, Essais sur l'espace, l'art et la destinée, Paris, Grasset, 1958, p. 36.

7. «L'œuvre acquit une qualité tactile. Elle favorisa ainsi la demande sur le marché cinématographique, car l'aspect distrayant du film a lui aussi en premier lieu un caractère tactile, en raison des changements de lieux et de plan qui assaillent le spectateur par à-coups. " (Walter Benjamin, L'œuvre d'art à l'époque de sa reproductibilité technique, Paris, Allia, 2003, p. 67.)

8. Thomas Sauels, Encountering Directors, New-York, Charles G.P. Putnams's Sons, 1972, p. 239, in Nathalie Bondil-Poupard, «Hitchcock, artiste malgré lui », Hitchcock et l'art : coïncidences fatales, sous la direction de Dominique Païni et Guy Cogeval, Milan, Centre Pompidou/Mazzotta, 2000, p. 184.

9. En avril 1948, John Cage et Merce Cunningham font une tournée à travers les États-Unis et passent par le Black Mountain College. Cunningham y a chorégraphié l'œuvre de Satie, le Piège de Méduse, ainsi que des morceaux de John Cage. La mise en scène est d'Helen Livingstone et Arthur Penn, les décors sont de Kooning. Parallèlement à John Cage, Cunningham y développe les procédures aléatoires et indéterminées de la danse.

10. Jacques Aumont, « Paradoxal et innocent » in Hitchcock et l'art, coïncidences fatales, op. cit. p. 95. 11. "Je dirai simplement, pour synthétiser, que le figural, matière de la pensée visuelle, relève davantage de la sensation que de la compréhension ou de la perception et qu'il est une catégorie difficile à traduire et même à appréhender, tant elle opère selon des modes non linéaires (comme le langage) et non transparents (comme l'image) mais plutôt selon les modes associatifs ouverts et multiples de la matière visuelle en elle-même, avec sa part d'irréductible opacité, qui fait sa force comme une tache aveugle est au fondement de l'activité de vision. » (Philippe Dubois, «La tempête et la matière-temps", in Jean Epstein, Cinéaste, poète, philosophe, sous la direction de Jacques Aumont, Paris, Cinémathèque Française, 1998, p. 270.)

12. John Dewey, L'art comme expérience, Paris, Gallimard, Folio/Essais, 2010, p. 179.

13. Siegfried Kracauer, Théorie du film, La rédemption de la réalité matérielle, Paris, Flammarion, Bibliothèque des savoirs, 2010, p. 416. Notons aussi que Kracauer, dans ce même ouvrage, rapproche cinéma et passage dans l'architecture de la ville moderne.

14. Henri Miller, Tropique du Cancer, Paris, Denoël, Folio, p. 356.

15. Roland Barthes, La Chambre Claire, Seuil/Cahiers du cinéma/Gallimard, 1980, p. 13.

16. Et la désignation de cette chose, légende, titre, texte, se fait l'indispensable guide à tout cheminement, toute aventure dans l'image, venant orienter, hiérarchiser, transformer le mouvement en sens. Et voilà que, lorsque tout n'est qu'itinérance programmée, politisée, le regard suit des routes toutes tracées et se fait aveugle, n'ayant pour lui aucun repère si ce n'est ce qu'il touche, ce qui lui tient lieu d'haptisme. Ou plutôt des repères, n'en dispose-t-il que trop, ce sont les cartels explicatifs dans une exposition venant coudre le regard à l'interprétation qu'on veut lui donner, ce sont les trames narratives dans le cinéma commercial qui nous font éluder la pauvreté de l'image vendue, distraction benjaminienne.

17. Luc Vancheri, Les pensées figurales de l'image, Paris, Armand Colin, 2011, p. 26.

18. Rosalind Krauss, Passages, une histoire de la sculpture de Rodin à Smithson, Paris, Macula, 1997, p. 277.

19. Pour la peinture, on pourra se reporter à la question du figural selon Deleuze analysant les polyptyques de Francis Bacon dans Logique de la sensation. [1981], Paris, Seuil, 2002. 


\section{RÉSUMÉS}

C'est au fil d'un aller-retour entre cinéma et arts plastiques que nous proposons d'envisager la possibilité d'un dessin filmique. Contrairement au dessin traditionnel visant à saisir les apparences et le miroitement du réel, la ligne cinématographique est un parcours pour l'œil et engage une image-sensation, le figural, qui semble résonner avec l'expérience que l'on a du monde des choses. Il en va d'un haptisme, d'un «toucher de l'œil». La Corde, pour un dessin filmique

\section{AUTEUR}

\section{DAPHNÉ LE SERGENT}

Maître de conférences à l'université Paris 8. Elle mène des recherches artistiques (vidéo/photo) et théoriques autour de la notion de schize et de frontière. Ce travail l'a conduite à réfléchir sur la question de l'agencement et du dispositif dans la création artistique contemporaine, présenté dans un ouvrage L'image-charnière ou le récit d'un regard (Paris, L'Harmattan, Ars, 2009). 